\title{
Synthesis of nano-carbon (nanotubes, nanofibres, graphene) materials
}

\author{
KALPANA AWASTHI, RAJESH KUMAR, HIMANSHU RAGHUBANSHI, \\ SEEMA AWASTHI, RATNESH PANDEY, DEVINDER SINGH, T P YADAV and \\ O N SRIVASTAVA* \\ Centre of Nanoscience and Nanotechnology, Department of Physics, Banaras Hindu University, \\ Varanasi 221005 , India
}

MS received 12 November 2009; revised 15 December 2009

\begin{abstract}
In the present study, we report the synthesis of carbon nanotubes (CNTs) using a new natural precursor: castor oil. The CNTs were synthesized by spray pyrolysis of castor oil-ferrocene solution at $850^{\circ} \mathrm{C}$ under an Ar atmosphere. We also report the synthesis of carbon nitrogen $(\mathrm{C}-\mathrm{N})$ nanotubes using castor oilferrocene-ammonia precursor. The as-grown CNTs and $\mathrm{C}-\mathrm{N}$ nanotubes were characterized through scanning and transmission electron microscopic techniques. Graphitic nanofibres (GNFs) were synthesized by thermal decomposition of acetylene $\left(\mathrm{C}_{2} \mathrm{H}_{2}\right)$ gas using Ni catalyst at $600^{\circ} \mathrm{C}$. As-grown GNFs reveal both planar and helical morphology. We have investigated the structural and electrical properties of multi-walled CNTs (MWNTs)-polymer (polyacrylamide (PAM)) composites. The MWNTs-PAM composites were prepared using as purified, with ball milling and functionalized MWNTs by solution cast technique and characterized through SEM. A comparative study has been made on the electrical property of these MWNTs-PAM composites with different MWNTs loadings. It is shown that the ball milling and functionalization of MWNTs improves the dispersion of MWNTs into the polymer matrix. Enhanced electrical conductivity was observed for the MWNTs-PAM composites. Graphene samples were prepared by thermal exfoliation of graphite oxide. XRD analysis confirms the formation of graphene.
\end{abstract}

Keywords. Carbon nanotube; graphitic nanofibres; graphene; CNTs-polymer composite; polyacrylamide.

\section{Introduction}

In the emerging field of nanomaterials, carbon nanostructures have potential significance. The various categories of carbon nanostructures include fullerenes, carbon nanotubes (CNTs), graphitic nanofibres (GNFs), graphene, etc. Carbon nanotubes (CNTs) have outstanding electrical, thermal and mechanical properties which make them interesting material for applications in nanoelectronics, sensors, field emission and as reinforcing agents in composite materials (Terrones 2003). The most important methods developed to produce CNTs include electric arc discharge (Ebbesen and Ajayan 1992), laser ablation (Thess et al 1996), and chemical vapor deposition (CVD) (Colomer et al 2000; Awasthi et al 2003). In any industrial application, an important issue in the production is the cost, quality, and yield of CNTs. The CVD method (in which hydrocarbon is pyrolyzed in the presence of transition metal catalysts) has attracted attention due to possibility to produce nanotubes on a commercial scale. Till date, several precursors such as carbon monoxide, methane, acetylene, benzene, xylene, etc have been used

*Author for correspondence (hepons@yahoo.com) as a carbon feedstock to synthesize CNTs (Srivastava et al 2004). These precursors are related to fossil fuels and they may be crisis for these precursors in near future. There are few reports on the synthesis of CNTs from natural precursors e.g. turpentine, eucalyptus oil and camphor (Afre et al 2006; Kumar and Ando 2005; Ghosh et al 2007). The advantages of using natural precursor as a carbon feedstock for synthesizing CNTs is that it is inexpensive and there is no chance of shortage in near future. Doping CNTs with heteroatom could be particularly an interesting way for tuning the surface and electronic properties. Incorporation of nitrogen in CNTs results in the enhancement of conductivity, due to the additional electron contributed by the nitrogen atom (Glerup et al 2003). The nitrogen containing carbon (C$\mathrm{N})$ nanotubes are effective field emitters.

CNTs based nanocomposites are increasingly being reviewed as a realistic alternative to conventional smart materials, as they offer higher sensitivity and superior electronic properties. The CNTs are expected to reinforce their unique properties to the polymer matrix with many further applications (Castro et al 2009). A number of research efforts have been used for polymer composites reinforcement (Awasthi et al 2006; Singh et al 2008). The optical and electrical properties of the poly 
(3-hexylthiophene) - SWNTs composites have been studied and an increase of $\sim 5$ orders of magnitude in electrical conductivity was observed for a $30 \mathrm{wt} \%$ concentration of SWNTs in polymer matrix (Singh et al 2008). But there are still many practical challenges in the preparation methods due to the agglomeration of CNTs. In order to optimally utilize the CNTs as a reinforcing component in the polymer matrix, some special treatments of CNTs, such as physical or chemical treatments are used to achieve better CNT dispersion and good interface between CNTs and polymer matrix (Moniruzzaman and Winey 2006). Physical methods involve ultrasonication, milling to physically separate the tubes that are bundled together. Chemical methods involve use of surfactant or chemical functionalization of the tube surface which improves interfacial bonding between the nanotubes and polymer and can prevent the tube aggregation. Several groups have studied the electrical and mechanical properties of CNTs-polymer composites using physically/ chemically treated CNTs (Ma et al 2007; Kim et al 2008). In our earlier study, we have prepared multiwalled CNTs (MWNTs)-polyethylene oxide (PEO) composites and studied their electrical and mechanical properties (Awasthi et al 2006). Enhanced electrical conductivity and mechanical strength were observed for the MWNTsPEO composites.

In other carbon nano materials such as graphite nanofibres (GNFs) graphitic sheets are parallel, perpendicular or inclined. Since the advent of GNFs (Baker et al 1972), they are being studied in depth due to their unique structure, distinctive physical and chemical properties and several feasible applications. GNFs can be used as reinforcement materials, battery components, polymer additives, fuel cell electrodes, nanoelectronics, hydrogen storage, novel catalyst, etc (De Jong and Gues 2000; Serp et al 2003; Nakayama 2004). GNFs are prepared by pyrolysing hydrocarbon compounds in the presence of transition metal particles like $\mathrm{Ni}, \mathrm{Co}, \mathrm{Fe}$ and alloys of these metal particles. The growth of GNFs has been achieved by employing carbon containing gases such as $\mathrm{CH}_{4}$, $\mathrm{C}_{2} \mathrm{H}_{6}, \mathrm{C}_{2} \mathrm{H}_{2}, \mathrm{C}_{2} \mathrm{H}_{4}, \mathrm{CO}$, etc used as feedstock. One exotic structure of the GNFs is its helical (coil) form known as helical graphitic (carbon) nano fibres. Carbon nanocoils have been synthesized (Pan et al 2002) by catalytic pyrolysis of acetylene using iron-coated indium tin oxide as catalyst. In this communication, we report synthesis of GNFs by employing fine particle of $\mathrm{Cu}-\mathrm{Ni}$ as catalyst.

New carbon materials such as graphene discovered recently are automatically thin sheets of carbon atoms tightly packed in a two dimensional honeycomb lattice, possessing many extraordinary properties, whose potential applications have recently been the subject of intense scientific interest (Geim and Novoselov 2007). The first historical isolation of single-layer graphene from the crystalline graphite was demonstrated by Novoselov et al (2004). Graphene exhibits fascinating properties such as quantum Hall effect at room temperature, ballistic conduction with high mean free path, tunable band gap, and high elasticity. Chemical and other properties of graphene also seem to be fascinating. Polymer composites based on graphene are expected to show interesting electronic and mechanical properties (Stankovich et al 2006). However, obtaining a single sheet of graphene is a challenge. Graphene was generally prepared by micromechanical cleavage of highly oriented pyrolytic graphite (HOPG) (Novoselov et al 2004). This method cannot be employed for large scale production or to study many of the chemical and mechanical properties of graphene. Various types of graphene samples have been prepared by chemical vapour deposition (Reina et al 2009), arc evaporation of $\mathrm{SiC}$ ( $\mathrm{Li}$ et al 2001), and exfoliation of graphite oxide (GO) (Schniepp et al 2003). Recently, graphene sheets were prepared by arc evaporation of graphite rod under hydrogen and argon atmosphere (Subrahmanyam et al 2009). More recently, graphene sheets (nanoribbons) were prepared by chemical oxidation of CNTs (Kosynkin et al 2009).

In the present study, we report the synthesis of CNTs/ $\mathrm{C}-\mathrm{N}$ nanotubes by spray pyrolysis, graphitic nanofibres by catalytic thermal decomposition of hydrocarbon gas such as $\mathrm{C}_{2} \mathrm{H}_{2}$. In addition, we also report the preparation of CNTs-polyacrylamide (PAM) composite films by solution cast technique by using as-purified, ball milled and functionalized MWNTs and synthesis of graphene sheets by thermal exfoliation of graphite oxide.

\section{Experimental}

\subsection{Synthesis of carbon/carbon-nitrogen $(C-N)$ nanotubes}

Synthesis of CNTs was carried out using spray pyrolysis assisted CVD method. We used ferrocene $\left(\mathrm{C}_{10} \mathrm{H}_{10} \mathrm{Fe}\right)$ as a source of iron $(\mathrm{Fe})$ which acts as a catalyst for the growth of CNTs. Castor oil was used as the carbon source. Castor oil contains carbon, hydrogen and lower amount of oxygen. The spray pyrolysis setup consisted of a nozzle (inner diameter $\sim 0.5 \mathrm{~mm}$ ) attached to a ferrocene-castor oil supply used for releasing the solution into a quartz tube $(700 \mathrm{~mm}$ long and inner diameter $25 \mathrm{~mm})$, which was mounted inside a reaction furnace $(300 \mathrm{~mm}$ long) (Srivastava et al 2004). The precursor solution i.e. ferrocene with castor oil (concentration $\sim 25 \mathrm{mg} / \mathrm{ml}$ ) was sprayed through the nozzle into the pre-heated $\left(850^{\circ} \mathrm{C}\right)$ quartz tube, using $\mathrm{Ar}$ as carrier gas. The optimum flow rate of Ar was $\sim 100 \mathrm{sccm}$ (standard cubic centimeter per minute). The precursor solution was sprayed for $15 \mathrm{~min}$ at a constant flow rate of $\sim 0.5 \mathrm{ml} / \mathrm{min}$. For $\mathrm{C}-\mathrm{N}$ nanotubes instead of castor oil and ferrocene solution, solution of castor oil-ferrocene with liquid ammonia $\left(\mathrm{NH}_{3}\right)$ was used. Ammonia was used to obtain native source of 
nitrogen in the precursor. The optimum ratio of castor oil to ammonia was $15: 1$ (volume wise). For this solution, the optimum ferrocene concentration was $20 \mathrm{mg} / \mathrm{ml}$. After deposition, the furnace was put off and allowed to cool down to room temperature under Ar gas flow. A black deposit was extracted from the reaction zone (center of the furnace) of quartz tube.

\subsection{Synthesis of graphitic nanofibres}

In the present investigation, GNFs were grown by using catalytic thermal decomposition of hydrocarbon gas such as $\mathrm{C}_{2} \mathrm{H}_{2}$. The $\mathrm{C}_{2} \mathrm{H}_{2}$ was prepared through the inexpensive process by employing calcium carbide $\left(\mathrm{CaC}_{2}\right)$ stone and water. Detailed description of the synthesis of GNFs are described in our previous publication (Singh et al 2004). Briefly, $100 \mathrm{mg}$ of $\mathrm{Ni}(96 \%)$ and $\mathrm{Cu}(4 \%)$ were taken for each run of the GNFs synthesis, since it was optimum for our experimental setup.

\subsection{Synthesis of CNTs-polymer (polyacrylamide) composites}

The MWNTs were functionalized with amino group via chemical modification of carboxylic groups introduced on the nanotube surface. This process involves a direct coupling of ethylenediamine with carboxylic groups to introduce amino groups via amide formation (Ramanathan et al 2005). The MWNTs-PAM composites were prepared by the solution cast technique using as-purified, ball-milled and functionalized CNTs. The details of the MWNTs-PAM composite preparation method is given in our previous publication. The PAM powder (BDH Chem. Ltd, Molecular weight $\sim 5 \times 10^{6}$ ) was dissolved in $50 \mathrm{ml}$ distilled water and mixed with MWNTs at different wt\% $(5,15,20,25,30$ and 40 (Awasthi et al 2009).

\subsection{Synthesis of graphene}

Graphite oxide (GO) was prepared by reacting graphite powder $(5 \mathrm{~g})$ with a mixture of conc. nitric acid $(45 \mathrm{ml})$ and sulphuric acid $(90 \mathrm{ml})$ with potassium chlorate $(\sim 55 \mathrm{~g})$ at room temperature for 5 days. For thermal exfoliation of graphite oxide, the dried graphite oxide powder $(\sim 200 \mathrm{~m})$ was placed in a quartz tube (diameter $\sim 25 \mathrm{~mm}$ and length $\sim 1.3 \mathrm{~m}$ ). The sample was flushed with Ar for $15 \mathrm{~min}$ and the quartz tube was quickly inserted into a furnace preheated to $1050^{\circ} \mathrm{C}$ and held in the furnace for $30 \mathrm{~s}$. The as-prepared GO was a brownish powder while the exfoliated version was of light consistency and shiny black.

The structural characterization of all the carbon nano materials were carried out using X-ray diffraction technique employing X'Pert PRO PANanalytical diffractometer equipped with graphite monochromator with $\mathrm{a} \mathrm{Cu}$ source $(\lambda=1.54 \AA, \mathrm{CuK} \alpha$ operating at $45 \mathrm{kV}$ and $40 \mathrm{~mA})$. The as-grown carboneous material was characterized by using scanning (SEM, Philips XL 20), and transmission (TEM, Tecnai $20 \mathrm{G}^{2}$ ) electron microscopes.

\section{Results and discussion}

\subsection{Carbon/carbon-nitrogen $(C-N)$ nanotubes}

Spray pyrolysis of castor oil-ferrocene solution at $\sim 850^{\circ} \mathrm{C}$ in $\mathrm{Ar}$ atmosphere leads to a uniform thick black deposition on the inner wall of the quartz tube at the reaction hot zone $\left(\sim 850^{\circ} \mathrm{C}\right)$. Figure la shows the SEM morphology of the as-grown CNTs. The length of CNTs is $\sim 5-10 \mu \mathrm{m}$. Structural details of the as-grown CNTs sample was further investigated by TEM. Typical TEM image of the as-grown CNTs is shown in Figure 1b. The TEM investigation of as-grown CNTs confirms that the CNTs are multi-walled in nature. These nanotubes have varying diameters ranging from $\sim 20-60 \mathrm{~nm}$. In the spray pyrolysis reaction, the castor oil-ferrocene solution was atomized via spray nozzle and sprayed through carrier gas (Ar). The Fe particles (liberated by the decomposition of ferrocene) were deposited on the inner walls of the quartz tube. The carbon species released from decomposition of castor oil and also from ferrocene got adsorbed on the $\mathrm{Fe}$ particles and diffused rapidly along the axial direction leading to the formation of CNTs.

In the present study we also used castor oil-ferrocene with ammonia solution so as to develop CNTs containing nitrogen i.e. $\mathrm{C}-\mathrm{N}$ nanotubes. This was done keeping in view the fact that nitrogen doped CNTs are considered as one of the important ingredients of CNT based electronics. Figure 2a shows a typical SEM micrograph of as-grown $\mathrm{C}-\mathrm{N}$ nanotubes, which reveals the wavy morphology of nanotubes. These wavy nanotubes are most likely due to pentagonal and heptagonal defects that are introduced in the hexagonal sheets. TEM images of the as-grown $\mathrm{C}-\mathrm{N}$ nanotubes are shown in figure $2 \mathrm{~b}$. These CNTs have bamboo-shaped structures. The TEM image (figure $2 \mathrm{~b})$ shows that the nanotubes have a range of diameters varying from $\sim 50-80 \mathrm{~nm}$. It is suggested that the bamboo-shaped morphologies arise from the incorporation of pyridine-like $\mathrm{N}$ atoms within the carbon framework (Nath et al 2000; Lee et al 2002). Also, no encapsulated metal particle was found inside the nanotubes. The tip of CNT is generally covered by catalyst particles.

\subsection{Graphitic nanofibres}

Average particle size of as-used $\mathrm{Cu}-\mathrm{Ni}$ catalyst was about $12 \mu \mathrm{m}$ measured by particle size analyzer which was also 


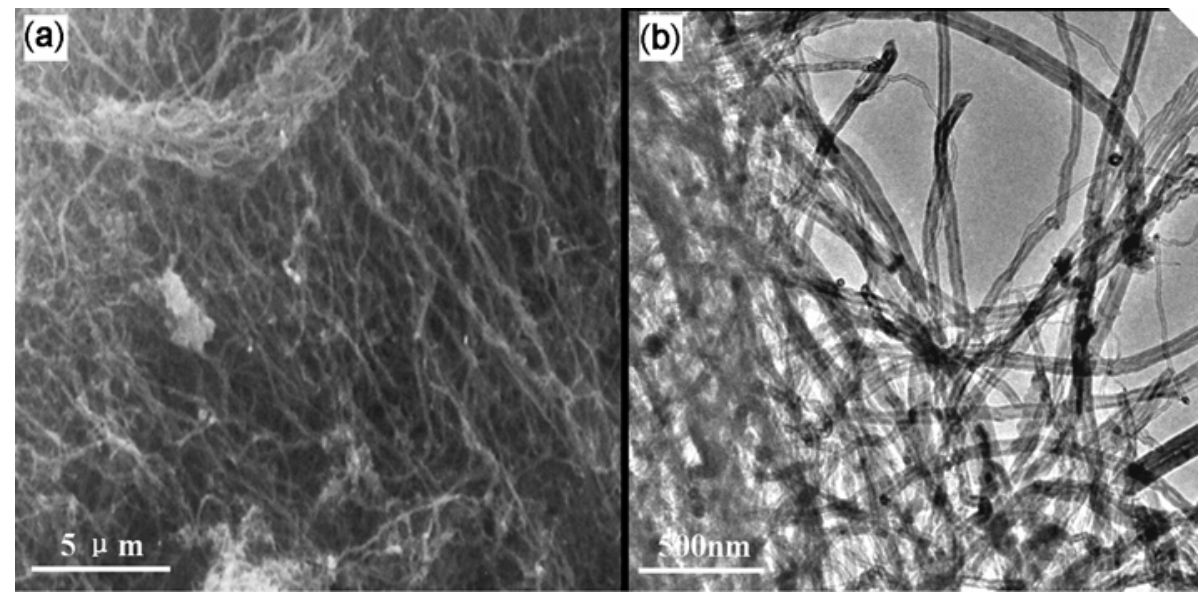

Figure1. SEM (a) and TEM (b) micrographs of the as-grown CNTs obtained by spray pyrolysis of castor oil-ferrocene solution at $\sim 850^{\circ} \mathrm{C}$.
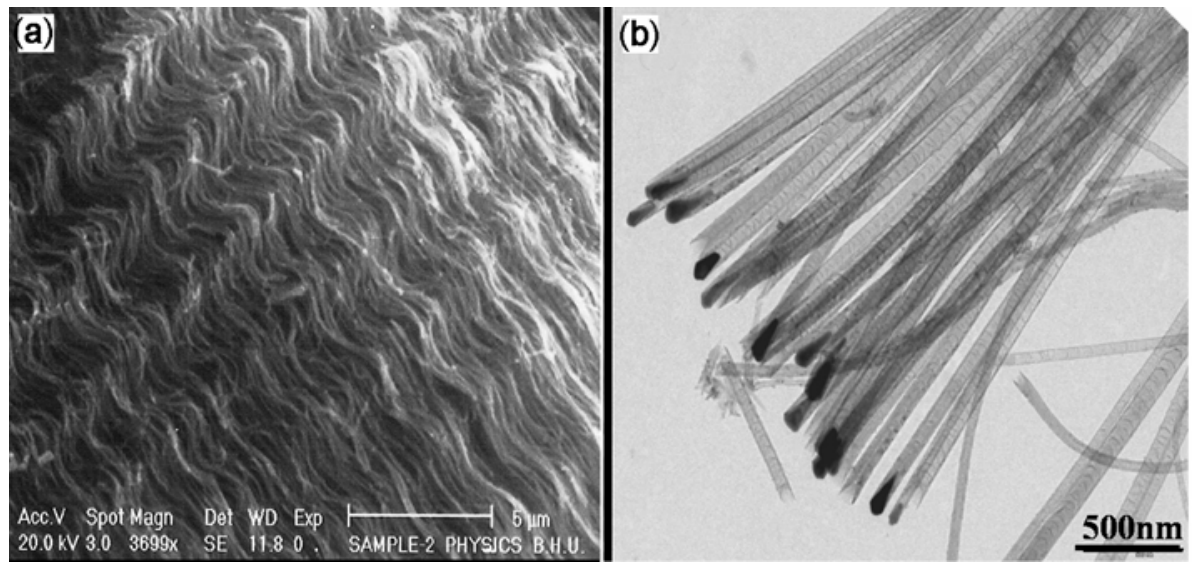

Figure 2. SEM (a) and TEM (b) micrographs of the as-grown $\mathrm{C}-\mathrm{N}$ nanotubes obtained by spray pyrolysis of castor oil-ferrocene with ammonia solution at $\sim 850^{\circ} \mathrm{C}$.

confirmed by TEM images. However, in the as-grown GNFs samples the size of the catalyst particles was in nanometer $(\sim 150 \mathrm{~nm})$ range which confirmed that at the time of GNFs synthesis these catalyst particles were broken into finer particles. After the growth of graphitic nanofibres, the as-synthesized product which also contained the catalyst particle was characterized through XRD. Analysis of the XRD pattern revealed the dominant presence of graphitic nano phase (crystallite size $3.6 \mathrm{~nm}$ ) together with nickel. The observed $d$ spacing of the GNFs was $\sim 0.342 \mathrm{~nm}$ which is very close to the ideal $d$ value of graphite $\sim 0.335 \mathrm{~nm}$. This shows that quite good graphitic nature is maintained for the synthesized GNFs. TEM micrographs shows that GNFs were synthesized successfully (figure 3 ). It was observed that at synthesis temperature $600^{\circ} \mathrm{C}$ the as-synthesized GNFs were in planar form (figure 3a). However, at synthesis temperature of $700^{\circ} \mathrm{C}$ the GNFs were in helical form (figure $3 b$ ). TEM explorations revealed that the $\mathrm{Ni}$ particle size associated with
GNF growth is $\sim 150 \mathrm{~nm}$ and also that it was equivalent to the diameter of the GNFs. Length of these GNFs were in the range of $6-8 \mu \mathrm{m}$ and the average diameter was $\sim 150 \mathrm{~nm}$.

It was observed that the morphology of the GNFs depend on the synthesis temperature. In the present investigation we have obtained both planar and helical morphology by using $\mathrm{Cu}-\mathrm{Ni}$ catalyst but at different temperature. Temperature is an important parameter which decides the catalyst shape according to which the morphology of the GNFs depends. The BET surface area of these GNFs was $207 \mathrm{~m}^{2} / \mathrm{g}$.

\subsection{CNTs-polymer (polyacrylamide) composites}

To study the surface morphologies of MWNTs-PAM composite films scanning electron microscopy studies were carried out. The SEM micrographs of MWNTs ( $\sim 5 \mathrm{wt} \%$ )-PAM composite film using purified and ball- 


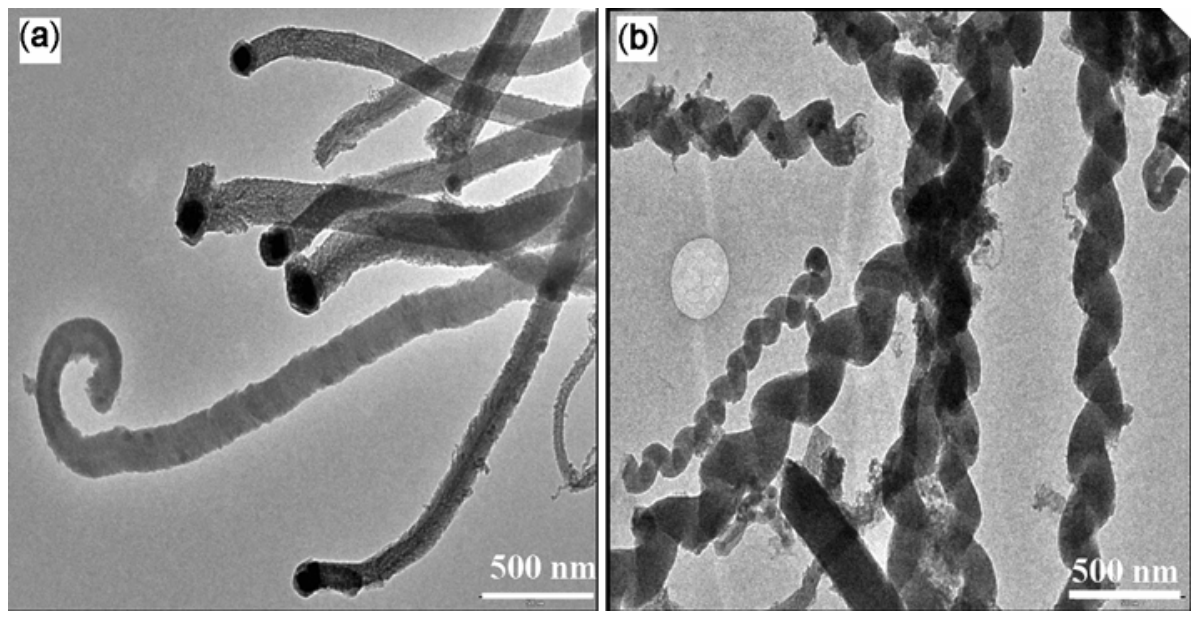

Figure 3. TEM images of GNFs synthesized by $\mathrm{Cu}-\mathrm{Ni}$ catalyst (a) reveals the planar form grown at $600^{\circ} \mathrm{C} ;(\mathbf{b})$ reveals the helical form grown at $700^{\circ} \mathrm{C}$.

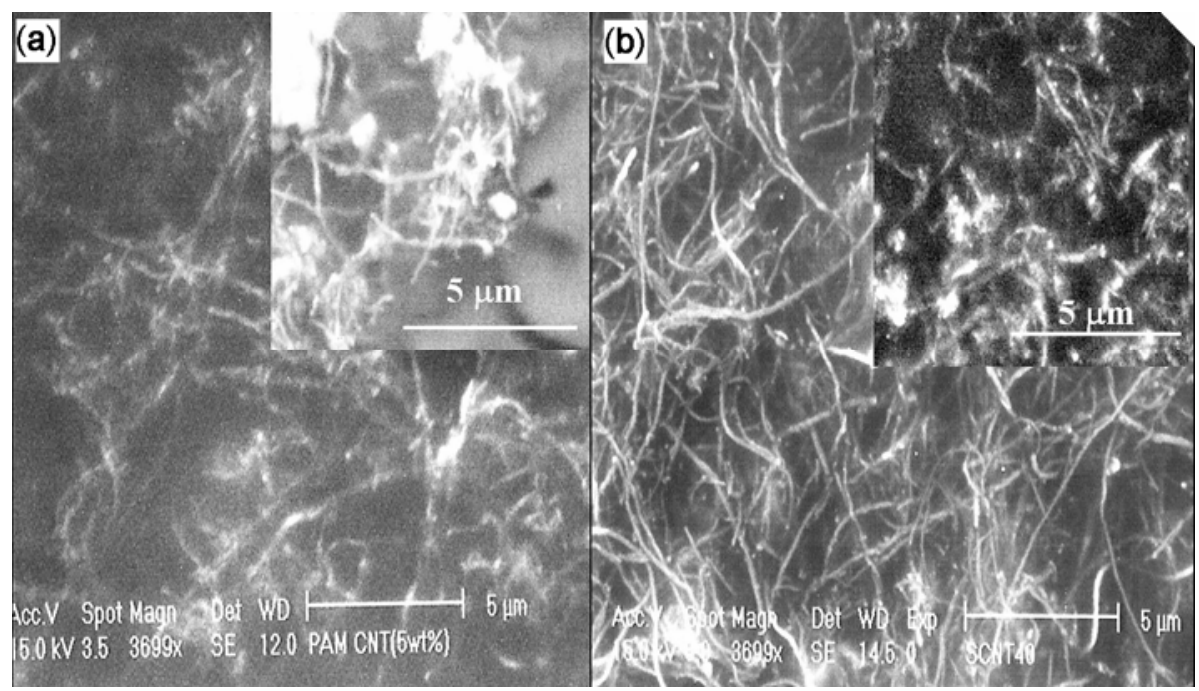

Figure 4. SEM micrographs of (a) MWNTs ( $~ 5 \mathrm{wt} \%)$ (ball-milled)-PAM composite film, Inset: MWNTs (purified)-PAM composite film. (b) MWNTs ( 40 wt \%))-PAM composite film Inset is for functionalized MWNTs ( $40 \mathrm{wt} \%)$-PAM composite film.

milled MWNTs is shown in figure 4a. The purified MWNTs (without ball-milling treatment) were present mainly in the form of agglomerates (inset of figure 4a), whereas the ball-milled MWNTs were dispersed more uniformly, confirming much better dispersion of MWNTs in PAM matrix. Figure $4 b$ shows SEM image of MWNTs (purified)-PAM composite with $40 \mathrm{wt} \%$ loading of nanotubes. The MWNTs were uniformly distributed throughout the polymer matrix.

To get a good dispersion for CNTs in matrix to receive a high interfacial bonding between CNTs and polymer matrix, it is necessary to attach some organic functional groups on the surface of CNTs. In the present investigation, amine group was attached onto the surface of MWNTs. Inset of figure $4 \mathrm{~b}$ shows the SEM images of
MWNTs ( $40 \mathrm{wt} \%)$-PAM composite films with amine functionalized MWNTs. The length of MWNTs decreased due to acid treatments in functionalization process. The electrical transport property (d.c. electrical conductivity) of the MWNTs-PAM composite was measured using the four-probe technique. The roomtemperature conductivity of the MWNTs-PAM composites at different loading of MWNTs is shown in figure 5. The d.c. electrical conductivity of pure PAM film was $\sim 1.05 \times 10^{-7} \mathrm{~S} / \mathrm{cm}$. No change in conductivity was observed at 5 and $10 \mathrm{wt} \%$ MWNTs (purified). The conductivity reaches $\sim 3.48 \times 10^{-6} \mathrm{~S} / \mathrm{cm}$ at $15 \mathrm{wt} \%$ MWNTs loading, which is one order of magnitude higher than that of PAM. At $40 \mathrm{wt} \%$ MWNTs loading, the conductivity of MWNTs-PAM composite was $\sim 1.2 \mathrm{~S} / \mathrm{cm}$. Thus the con- 
ductivity of MWNTs ( $40 \mathrm{wt} \%)$-PAM composite increases by seven orders of magnitude.

The conductivity increase with MWNTs content fraction (wt\%) indicates a typical percolation behavior. The MWNTs (ball-milled) ( $5 \mathrm{wt} \%)-P A M$ composite film shows increase in conductivity by three orders of magnitude higher than pure PAM film. The significant increase in conductivity suggests that dispersion of nanotubes was increased due to ball-milling process. The electrical conductivity of functionalized MWNTs-PAM composite film was the same as that of pure PAM at 5 and $15 \mathrm{wt} \%$ MWNTs loadings. At 20 and $40 \mathrm{wt} \%$ functionalized MWNTs loading, the conductivity was three and four orders of magnitude higher than pure PAM film. The decrease in electrical conductivity of MWNTs

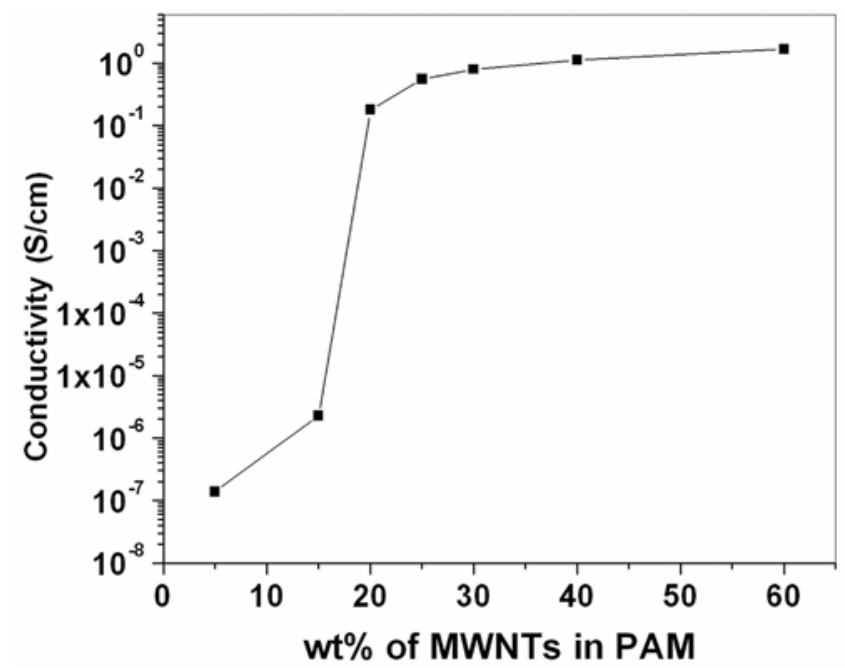

Figure 5. Room temperature electrical conductivity of MWNTs-PAM composite film vs the MWNTs loading (wt \%).

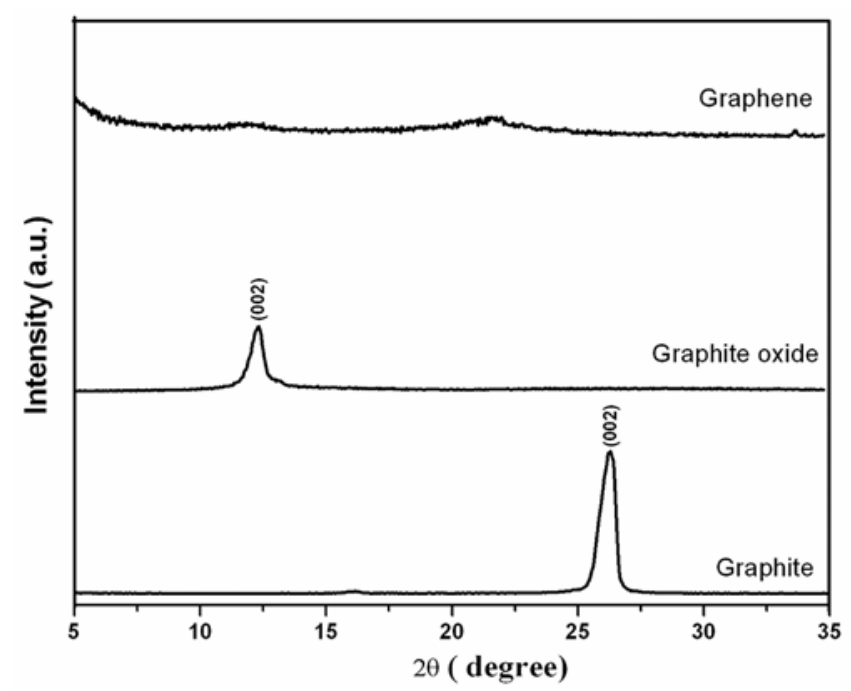

Figure 6. XRD patterns of graphite, graphite oxide and graphene samples.
(functionalized)-PAM composite as compared to MWNTs (purified)-PAM was expected. The electrical conductivity decreased due to the presence of functional group on the surface of CNTs. Further investigations regarding mechanical properties of MWNTs-PAM composites are being carried out and results will be forthcoming.

\subsection{Graphene}

A definitive identification of graphene can be made by analysis of XRD pattern. The XRD pattern of graphite, graphite oxide and graphene are shown in figure 6. XRD pattern of graphite shows an intense peak $2 \theta=26.4^{\circ}$. This peak corresponds to 002 plane of graphite with interlayer spacing of $0.34 \mathrm{~nm}$. In the XRD pattern of graphite oxide a new peak appears at $2 \theta=13 \cdot 2^{\circ}$, corresponding to the 002 plane of graphite oxide (Schniepp et al 2003). The interlayer spacing of GO is $\sim 0.75 \mathrm{~nm}$, which is significantly larger than that of graphite, due to intercalating oxide functional groups. The mechanism of exfoliation is mainly the expansion of $\mathrm{CO}_{2}$ evolved into the interstices between the graphene sheets during rapid heating. The disappearance of native graphite XRD peaks in the XRD pattern of as-prepared graphene sample supports the formation of graphene sheets. The SEM image of graphene sample is shown in figure $7 a$, where the sheets are highly agglomerated and particles have a fluffy morphology. The TEM image of graphene sample shows a wrinkled paper like structure in low magnification. The selected area diffraction (SAED) pattern of graphene sheets is shown in inset of figure $7 \mathrm{~b}$. The diffraction pattern indicates the formation of few layers of graphene.

We have measured the d.c. electrical conductivity of as-prepared graphene sample by four probe setup which was $\sim 10.6 \mathrm{~S} / \mathrm{cm}$ at room temperature. The characterization of graphene sample and investigation of graphenepolymer composites are being carried out and results will be forthcoming.

\section{Conclusions}

Based on the present investigations, the following conclusions can be drawn:

(I) Multi-walled CNTs were prepared in high yield by spray pyrolysis of castor oil-ferrocene solution at $850^{\circ} \mathrm{C}$ under Ar atmosphere. The $\mathrm{C}-\mathrm{N}$ nanotubes were synthesized by modifying the castor oil-ferrocene precursor with ammonia at $850^{\circ} \mathrm{C}$. The morphology of the as-grown CNTs and $\mathrm{C}-\mathrm{N}$ nanotubes has been verified by SEM and TEM. The advantage of the present synthesis technique is the simplicity and use of low cost and easily available precursors. 


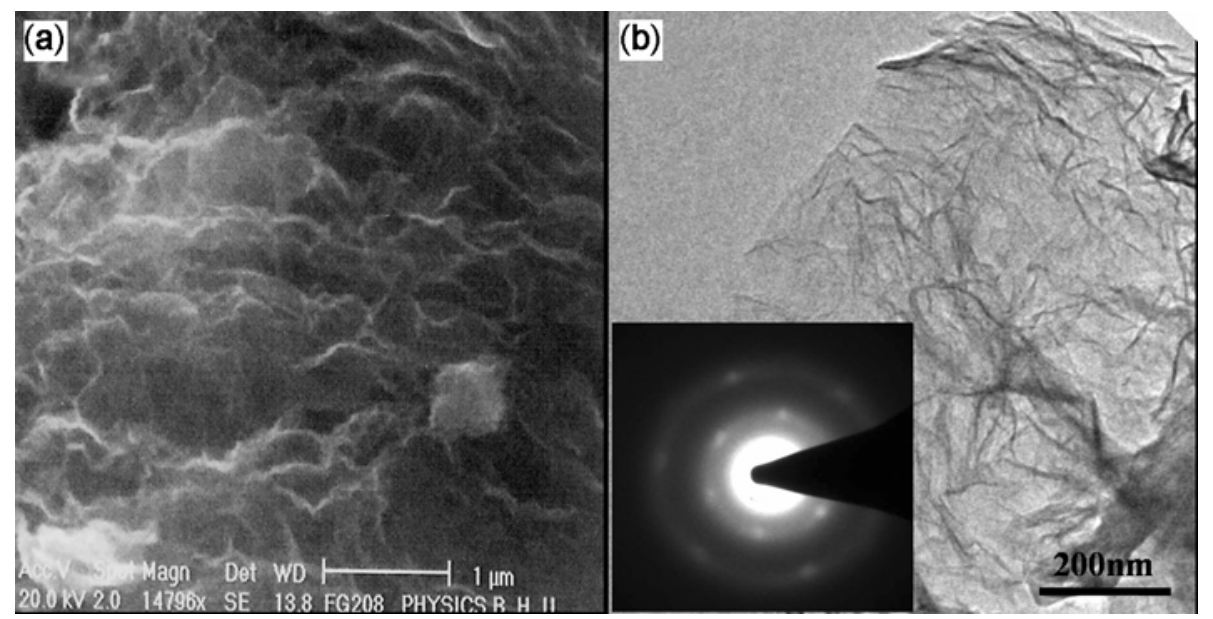

Figure 7. SEM (a) and TEM (b) images of graphene samples. Inset of (b) shows the SAED pattern of graphene sample.

(II) Graphitic nanofibres (GNFs) were successfully synthesized by employing fine particles of $\mathrm{Cu}-\mathrm{Ni}$ as a catalyst. Catalyst assisted thermal decomposition of hydrocarbon gas method was used for the synthesis of GNFs at two different temperatures, $600^{\circ} \mathrm{C}$ and $700^{\circ} \mathrm{C}$, by using $\mathrm{H}_{2}$ as carrier gas and $\mathrm{C}_{2} \mathrm{H}_{2}$ as a feedstock gas. Microstructural analysis shows that the planar GNFs are formed at $600^{\circ} \mathrm{C}$ and helical GNFs at $700^{\circ} \mathrm{C}$. BET analysis shows that these GNFs possessed high surface area (207 $\left.\mathrm{m}^{2} / \mathrm{g}\right)$.

(III) We have prepared MWNTs-PAM composite films using as-purified, ball-milled and functionalized MWNTs. The effect of ball-milling and functionalization of CNTs on the properties (dispersion, electrical conductivity) of composites have been studied. The present investigations revealed that in PAM film, conductivity at room temperature is $1.05 \times 10^{-7} \mathrm{~S} / \mathrm{cm}$ and for MWNT ( $40 \mathrm{wt} \%$ )-PAM film, it increases to $1.2 \mathrm{~S} / \mathrm{cm}$ i.e. by seven orders of magnitude. SEM micrographs show that nanotubes dispersion improved through ball-milling of MWNTs at low nanotubes loading ( $5 \mathrm{wt} \%)$. Functionalization of nanotubes can improve the interfacial bonding between the nanotubes and polymer matrix. But electrical conductivity decreased due to attachment of functional group onto CNT surface.

(IV) We have successfully synthesized graphene sheets by thermal exfoliation of graphite oxide at $1050^{\circ} \mathrm{C}$ under Ar atmosphere. XRD pattern of as-prepared graphene sample did not present any diffraction peak indicating the formation of graphene. The TEM images reveal a wrinkled paper like structure.

\section{Acknowledgements}

The authors are grateful to the Nanoscience and Technology Initiative Department of Science and Technology
(DST), India for the financial support. We would also like to thanks Prof. C N R Rao, Prof. A K Raychoudhary, Prof. D P Singh and Prof. S Lele. The financial supports from CSIR, UGC, MNRE, New Delhi and DST (UNANST: BHU) are also gratefully acknowledged.

\section{References}

Afre R A, Soga T, Jimbo T, Kumar M, Ando Y, Sharon M, Prakash R S and Umeno M 2006 Micro. Mesop. Mat. 96 184

Awasthi K, Awasthi S, Srivastava A, Kamalakaran R, Talpatra S, Ajayan P M and Srivastava O N 2006 Nanotechnology 17 5417

Awasthi S, Awasthi K, Kumar R and Srivastava O N 2009 J. Nanosci. Nanotechnol. 95455

Baker R T K, Barber M A, Harris P S, Feates F S and Waite R J 1972 J. Catal. 2651

Castro M, Lu J, Bruzaud S, Kumar B and Feller J 2009 Carbon 471930

Colomer J.F, Stephan C, Lefrant S, Tendeloo G Van, Willems I, Konya Z, Fonseca A, Laurent C and Nagy J B 2000 Chem. Phys. Lett. 31783

De Jong K P and Geus J W 2000 Cat. Rev .Sci. Eng. 42 481

Ebbesen T W and Ajayan P M 1992 Nature 358220

Geim A K and Novoselov K S 2007 Nature Mater. 6183

Ghosh P, Afre R A, Soga T and Jimbo T 2007 Mat. Lett. 61 3768

Glerup M, Castignolles M, Holzinger M, Hug G, Loiseau A and Bernier P 2003 Chem. Commun. 2542

Kaufman L and Nestor H 1978 Calphad 2325

Kim H S, Chae Y S, Park B H, Yoon J S, Kang M and Jin H J 2008 Curr. Appl. Phys. 8803

Kosynkin D V, Higginbotham A L, Sinitskii A, Lomeda J R, Dimiev A, Price B K and Tour J M 2009 Nature 458872

Kumar M and Ando Y 2005 Carbon 43533

Lee C J, Lyu S C, Kim H W, Lee J H and Cho K I 2002 Chem. Phys. Lett. 359115 
Li Y, Xie S, Zhou W, Tang D, Zou X P, Liu Z and Wang G, 2001 Carbon 39626

Ma P C, Kim J and Tang B Z 2007 Compos. Sci. Technol. 67 2965

Moniruzzaman M and Winey K I 2006 Macromolecules 39 5194

Nakayama Y 2004 Function Mater. 2414

Nath M, Satishkumar B C, Govindraj A, Vinod C P and Rao C N R 2000 Chem. Phys. Lett. 322333

Novoselov K S, Geim A K, Morozov S V, Jiang D, Zhang Y, Dubonos S V, Grigorieva I V and Firsov A A 2004 Science 306666

Pan L, Zhang M and Nakayama Y 2002 J. Appl. Phys. 91 10058

Ramanathan T, Fisher F T, Ruoff R S and Brinson L C 2005 Chem. Mater. 171290

Reina A, Jia, X, Ho J, Nezich D, Son H, Bulovic V and Dresselhaus M S Kong 2009 Nano Lett. 930
Schniepp H C et al 2006 J. Phys. Chem. B110 8535

Serp P, Corrias M and Kalck P 2003 Appl. Catal. A: General 253337

Singh I, Bhatnagar P K, Mathur P C, Kaur I, Bharadwaj L M and Panday R 2008 Carbon 461141

Srivastava A, Srivastava O N, Talapatra S, Vajtai R and Ajayan P M 2004 Nature Mater. 3610

Stankovich S et al 2006 Nature 442282

Subrahmanyam K S, Panchakarla L S, Govindaraj A and Rao C N R 2009 J. Phys. Chem. C113 4257

Tcherdyntseva V V, Kaloshkina S D, Shelekhova E V, Salimona A I, Sartorib S and Principi G 2005 Intermetallics 13 841

Terrones A 2003 Annu. Rev. Mater. Res. 33419

Thess A, Lee R, Nikolaev P, Dai H, Petit P, Robert J, Xu C, Hee Y, Kim S G, Rinzler A G, Colbert D T, Scuseria G E, Tománek D, Fischer J E and Smalley R 1996 Science 273 483 